\title{
IX. Ventilación no invasiva en el paciente con enfermedad pulmonar obstructiva crónica avanzada estable
}

\author{
CARLOS INZUNZA P.* y ORLANDO DÍAZ P.**
}

\author{
IX. Noninvasive ventilation in patients with severe chronic obstructive \\ pulmonary disease
}

La ventilación no invasiva (VNI) en el paciente con enfermedad pulmonar obstructiva crónica (EPOC) e insuficiencia respiratoria aguda es ampliamente aceptada ${ }^{1,2}$. No ha sucedido lo mismo en la EPOC avanzada estable, condición en la que su empleo ha sido muy debatido y la evidencia disponible no es absolutamente convincente.

La creciente utilización domiciliaria de la VNI, basada en indicaciones cuestionables, y para una serie de condiciones entre las que se incluye la EPOC, forzó a un consenso norteamericano en $1999^{3}$. Las recomendaciones allí sugeridas continúan utilizándose pero son algo diferentes a las enunciadas posteriormente por el British Medical Council ${ }^{4}$, lo que refleja principalmente la ausencia de información que las avale.

Gran parte de la controversia se debe a que, a diferencia de otras áreas en que la VNI se aplica, en estos pacientes todavía existen muchas preguntas no aclaradas. Así, por ejemplo, no está definido a quiénes eventualmente debería aplicarse (características clínicas); cómo debería aplicarse (parámetros del ventilador); cuándo debería aplicarse (día o noche); qué debería esperarse de su aplicación (efectos sobre la función respiratoria; disnea; capacidad de ejercicio; calidad del sueño; calidad de vida; exacerbaciones; hospitalizaciones; mortalidad). Consecuentemente, el actual documento se presentará de una manera diferente a otros de este Consenso, en los que el tipo de paciente y los efectos esperados se conocen.

\section{Mecanismos de acción}

Existen tres hipótesis respecto a los mecanismos por los cuales la VNI podría ejercer un efecto beneficioso sobre las alteraciones fisiológicas y los síntomas de la EPOC avanzada mencionados anteriormente. Una de ellas plantea que estos efectos se deben al reposo de los músculos respiratorios, aliviándolos de un eventual estado de fatiga crónica; otra, a una mejoría de las características del sueño, particularmente de la hipoventilación nocturna; y una tercera, a la reducción de la hiperinflación pulmonar.

Hipótesis de la fatiga muscular inspiratoria. Cuando se inició el empleo de la VNI en este grupo de pacientes se había planteado la hipótesis que en la EPOC avanzada asociada a hipercapnia existía un estado de fatiga crónica de los músculos inspiratorios ${ }^{5}$. De acuerdo a esta propuesta, períodos de reposo intermitente recuperaban la función muscular, traduciéndose en un aumento de la fuerza muscular inspiratoria y una disminución de la $\mathrm{PaCO}_{2}{ }^{6-10}$. Se observó que concomitantemente disminuía la disnea, aumentaba la capacidad de ejercicio ${ }^{6,10}$ y mejoraba la calidad de vida ${ }^{9}$. Sin embargo, ensayos clínicos controlados posteriores no confirmaron los cambios en la función muscular ${ }^{11-13}$ y estudios adicionales han puesto en duda incluso que exista este estado de fatiga crónica. Ello se debe a que al comparar índices de función muscular inspiratoria de pacientes con EPOC avanzada y sujetos

\footnotetext{
* Servicio de Medicina, Instituto Nacional del Tórax.

** Profesor Asociado de Medicina, Departamento de Enfermedades Respiratorias, Pontificia Universidad Católica de Chile.
} 
sanos a volúmenes pulmonares equivalentes, ésta es ligeramente superior en los pacientes ${ }^{14}$. Por tal razón, se ha postulado que la reducción de la fuerza muscular inspiratoria de la EPOC es principalmente un efecto secundario a la hiperinflación pulmonar, mientras que la hipercapnia correspondería a una estrategia del sistema respiratorio frente al aumento de la carga inspiratoria y a la disminución de la fuerza muscular propia de la enfermedad avanzada ${ }^{15}$. En este escenario, el centro respiratorio aparentemente reduce la ventilación alveolar para disminuir la sobrecarga muscular inspiratoria y por lo tanto impide una eventual fatiga.

Hipótesis del sueño. Una hipótesis alternativa surgió posteriormente, producto del estudio de las características del sueño en la EPOC ${ }^{16,17}$. Estas investigaciones demostraron una elevada prevalencia de trastornos respiratorios nocturnos en pacientes con enfermedad avanzada, que no sólo incluían apneas obstructivas, sino que también episodios de hipoventilación e hipopneas ${ }^{17}$. Como consecuencia, la arquitectura del sueño estaba afectada por fragmentación y disminución de los períodos de sueño $\mathrm{REM}^{16}$. Se ha planteado que la VNI usada durante la noche prevendría tales trastornos, reduciendo los microdespertares asociados, y mejorando la calidad del sueño. Asimismo, la menor hipoventilación evitaría la retención nocturna de $\mathrm{CO}_{2}$ y el consecuente aumento del bicarbonato sérico. El efecto de estos cambios sobre el centro respiratorio debería ser un aumento de su sensibilidad al $\mathrm{CO}_{2} \mathrm{y}$, por lo tanto, un incremento del estímulo para la ventilación ${ }^{18-20}$. Según esta hipótesis, el efecto neto de la VNI se traduciría en una mejoría de la $\mathrm{PaCO}_{2}$ diurna, de la arquitectura del sueño y de la calidad de vida. Como la hipercapnia también afecta la función de los músculos inspiratorios ${ }^{21}$ y posee un efecto disneogénico $^{22}$, su corrección mediante la VNI podría explicar al menos en parte el aumento de la fuerza muscular inspiratoria, la reducción de la disnea y la mayor capacidad de ejercicio observada con el empleo de VNI. Aunque cinco estudios controlados evaluaron formalmente el efecto de la VNI sobre las características del sueño ${ }^{19,23-26}$, sólo uno de ellos ha demostrado hasta ahora una clara mejoría de su arquitectura ${ }^{19}$.

Hipótesis de la reducción de volumen pulmonar. La asociación entre hipercapnia e hiperinflación pulmonar es ampliamente conocida ${ }^{15,27}$. Se ha observado que la aplicación de VNI prolonga el tiempo espiratorio, lo que podría permi- tir el vaciamiento de unidades alveolares lentas y sobredistendidas, y el reclutamiento de unidades vecinas colapsadas, pero funcionalmente normales, aumentando los flujos espiratorios ${ }^{28}$. Estos efectos fisiológicos, similares a los que provoca la cirugía de reducción de volumen pulmonar ${ }^{29}$, conducirían a una disminución de la hiperinflación pulmonar, a un incremento de la fuerza muscular respiratoria debido a la reposición del diafragma y a una disminución de la carga inspiratoria elástica. De acuerdo a esta teoría, las consecuencias clínicas esperables de la VNI deberían incluir una reducción de la disnea y la $\mathrm{PaCO}_{2}$, un aumento de la capacidad de ejercicio y una mejor calidad de vida. Este mecanismo, propuesto años atrás en un estudio no controlado $^{18}$ ha sido recientemente confirma$\mathrm{do}^{28,30,31}$.

La importancia de estas hipótesis no es menor, ya que pueden influir en la forma como se administra la VNI. Por ejemplo, para reposar los músculos inspiratorios se ha sugerido que la VNI debería aplicarse al menos 5 horas diarias ${ }^{32}$ y que bastarían presiones inspiratorias bajas $(\sim 10$ $\mathrm{cm} \mathrm{H}_{2} \mathrm{O}$ ) para reducir la actividad diafragmática $^{33}$. En cambio, para mejorar las características del sueño la aplicación debería ser nocturna, mientras que para remover la $\mathrm{PaCO}_{2}$ provocada por la hipoventilación del sueño las presiones inspiratorias deberían oscilar entre 15-20 $\mathrm{cm} \mathrm{H}_{2} \mathrm{O}^{34}$. Por último, para reducir la hiperinflación pulmonar la aplicación puede ser diurna o nocturna, se ha observado que puede modificarse con aplicaciones de sólo 3 horas ${ }^{28}$, y la reducción es directamente proporcional a la presión inspiratoria, ${ }^{33}$ por lo que se requerirían presiones elevadas.

\section{Evidencia clínica}

Varias razones probablemente expliquen la falta de consistencia en los resultados de los diferentes estudios publicados. Para analizar las posibles causas se evaluaron los ensayos clínicos randomizados (ECR) publicados, tanto aquellos en que se aplicó presión negativa o positiva, durante el día o la noche, e independientemente de su duración. Debido a que la duración es muy variable, arbitrariamente los estudios se han diferenciado en aquellos de corto ( $\leq 1 \mathrm{mes})$, intermedio (1-12 meses) y largo plazo ( $\geq 1$ año). Los hallazgos de las revisiones sistemáticas y meta-análisis son discutidos al final.

Hasta la fecha se encontraron 19 estudios (2 de diseño cruzado) con una duración que varía 
entre 3 días y 2 años $6,8,10-13,19,23-26,28,30,35-40$. Descartamos cinco que sólo evaluaron cambios fisiológicos ${ }^{8,10,28,36,38}$. Un estudio adicional no fue considerado porque la VNI se empleó en concomitancia con entrenamiento físico, y no fue ciego para pacientes ni evaluadores, resultando difícil separar los efectos de las dos intervenciones $^{35}$. De los 13 estudios seleccionados, cinco fueron realizados con ventiladores de presión negativa, siendo dos de ellos de duración intermedia y el resto de corto plazo. En otros ocho ECR se empleó ventiladores de presión positiva, 3 de los cuales fueron de corta duración, 3 de duración intermedia y 2 de largo plazo. Todos los ventiladores de presión positiva han sido equipos portátiles que han empleado la modalidad binivel, usando como interfase una mascarilla nasal, excepto en dos estudios en los que se usaron mascarillas oro-nasales ${ }^{28,30}$.

Las características de los estudios se proporcionan en las Tablas 1-4. Se ha incluido los valores de $\mathrm{PaCO}_{2}$ de los pacientes del grupo tratado $\mathrm{y}$, en los estudios en que se utilizó presión inspiratoria positiva, se describen sus niveles.

Efectos evaluados. Aunque todos estos ECR han estudiado el impacto de la VNI sobre el intercambio de gases, los beneficios de mayor relevancia para el paciente se han evaluado de manera menos sistemática. Estos efectos clíni- cos han incluido la disnea, empleando diferentes escalas $6,23,24,26,30,37,39,40$; la capacidad de ejercicio, principalmente mediante la caminata en 6 ó 12 minutos $^{6,19,23,30,37,39}$, la calidad de vida ${ }^{12,19,23}$; las exacerbaciones $^{23}$; y la mortalidad ${ }^{23,40}$. En algunos casos se exploraron también los efectos sobre las características del sueño ${ }^{19,25}$. Para ser concisos, en adelante generalmente se hará referencia a estos estudios como favorables o negativos, según si han tenido o no algún efecto clínicamente significativo en estas variables.

Ventiladores de presión negativa. La Tabla 1 describe los cinco estudios publicados. Uno de los aspectos destacables es que la $\mathrm{PaCO}_{2}$ promedio de aquellos con resultados negativos ${ }^{11-13}$ resulta notoriamente inferior a la de aquellos con resultados favorables ${ }^{6,37}$ (45 vs $59 \mathrm{mmHg}$, respectivamente). Asimismo, los dos trabajos favorables fueron realizados en el hospital, bajo estrecha supervisión, mientras que dos de los estudios negativos se efectuaron en el domicilio, sin vigilancia ${ }^{12,13}$. Otro hecho que destaca es la alta tasa de abandonos. Especial mención merece el estudio de Shapiro y cols ${ }^{12}$, pues incorporó el mayor número de pacientes comunicado hasta ahora $(\mathrm{n}=184), 92$ de los cuales fueron asignados a recibir VNI domiciliaria. Debido a la ausencia de efectos clínicos en el grupo tratado y a que la $\mathrm{PaCO}_{2}$ promedio fue sólo $44 \pm 7 \mathrm{mmHg}$,

Tabla 1. Ventilación no invasiva con ventiladores de presión negativa. Estudios de corto y mediano plazo

\begin{tabular}{lllcc}
\hline Autores (Ref) & Diseño & Duración & $\begin{array}{c}\text { Número } \\
\text { Inician/Completan }\end{array}$ & PaCO $_{\mathbf{2}} \mathbf{~ m m H g}^{\dagger}$ \\
\hline Zibrack, $1988^{13}$ & Cruzado & $4-6$ meses & $20 / 9$ & $47 \pm 13$ \\
Celli, $1989^{11}$ & Paralelo & 3 semanas & $9 / 8$ & $45 \pm 7$ \\
Shapiro, $1992^{12}$ & Paralelo & 3 meses & $92 / 58$ & $44 \pm 7$ \\
Ambrosino, $1990^{6}$ & Paralelo & 5 días & $10 / 10$ & $56 \pm 4$ \\
Gigliotti, $1994^{37}$ & Paralelo & 4 semanas & $6 / 6$ & $62 \pm 6$ \\
\hline
\end{tabular}

† Sólo se proporcionan los datos del grupo tratado con ventilación no invasiva. (Ref) = cita bibliográfica.

Tabla 2. Ventilación no invasiva con ventiladores de presión positiva. Estudios de corto plazo

\begin{tabular}{llllcc}
\hline Autores (Ref) & $\begin{array}{c}\text { Presión, } \\
\text { cm } \mathbf{H}_{\mathbf{2}} \mathbf{O}^{*}\end{array}$ & Diseño & Duración & $\begin{array}{c}\text { Número } \\
\text { Inician/Completan }\end{array}$ & $\begin{array}{c}\text { PaCO}_{\mathbf{2}}, \\
\mathbf{m m H g}^{\dagger}\end{array}$ \\
\hline Lin, $1996^{25}$ & $12 / 2$ & Cruzado & 2 semanas & $12 / 12$ & $51 \pm 4$ \\
Renston, $1994^{39}$ & $15-20 / 2$ & Paralelo & 5 días & $9 / 9$ & $\sim 50$ \\
Díaz, $2005^{28}$ & $18 \pm 2 / 2$ & Paralelo & 3 semanas & $18 / 18$ & $57 \pm 6$ \\
\hline
\end{tabular}

* Se describe presión inspiratoria/presión espiratoria. (Ref) = cita bibliográfica.

†Sólo se proporcionan los datos del grupo tratado con ventilación no invasiva. 
los autores efectuaron un análisis de subgrupos usando como punto de corte una $\mathrm{PaCO}_{2}$ de 45 mmHg. Los resultados en los subgrupos fueron igualmente negativos. Más que descartar la posible utilidad de la VNI, este ECR demostró la pobre tolerancia a la VNI domiciliaria cuando se emplean ventiladores de presión negativa, ya que 63 de los 92 pacientes tratados no lo usaron apropiadamente y 34 nunca lo usaron o abandonaron el estudio precozmente.

\section{Ventiladores de presión positiva en estudios} de corto plazo. Se han publicado 3 ECR cuya duración ha oscilado entre 5 días y tres sema-

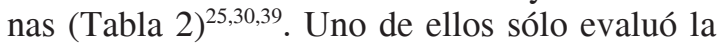
eficiencia del sueño, la que empeoró con $\mathrm{VNI}^{25}$. Debido a su duración de apenas 2 semanas, se ha sugerido que estos pacientes no tuvieron tiempo suficiente para aclimatarse al respirador. Los otros dos estudios, con una duración de 5 días ${ }^{39}$ y 3 semanas $^{30}$, fueron realizados en laboratorios de función pulmonar durante el día y bajo estrecha vigilancia, y comunicaron un aumento de la capacidad para caminar y una reducción de la disnea. Sin embargo, en uno de ellos se observó un efecto similar aunque de menor magnitud en el grupo control y los autores no especifican si las diferencias entre ambos grupos fueron significativas $^{39}$. Estos estudios sugieren que la estrecha supervisión de la VNI puede ser un factor importante en el éxito de la intervención, pero poseen el inconveniente de no permitir evaluar la tolerancia a largo plazo del tratamiento.

Ventiladores de presión positiva en estudios de mediano plazo. Tres estudios de duración intermedia han sido publicados, los que se resumen en la Tabla $3^{19,24,26}$.

Dos ECR de esta categoría poseen igual diseño (cruzados, tres meses de duración), pero resultados opuestos, por lo que frecuentemente se han comparado en otras revisiones. El estudio de Meecham-Jones y cols ${ }^{19}$, de efectos favorables, incluyó 18 pacientes hipercápnicos, de los cuales 14 completaron el estudio. La VNI nocturna más oxigenoterapia domiciliaria fue comparada con oxigenoterapia sola, observándose una reducción significativa de la $\mathrm{PaCO}_{2}, \mathrm{y}$ una mejoría de la calidad del sueño y de la calidad de vida al aplicar VNI. Se observó asimismo un aumento no significativo de la distancia recorrida en 6 minutos. Por el contrario, los resultados de Strumpf y cols ${ }^{26}$, fueron desfavorables. Al igual que en el estudio previo, se comparó VNI nocturna con tratamiento estándar. De los 23 participantes incluidos sólo 7 completaron las dos ramas del protocolo, en gran parte debido a abandonos por intolerancia. No se demostró mejoría del intercambio gaseoso, disnea, función neuro-psicológica ni calidad del sueño.

Tabla 3. Ventilación no invasiva con ventiladores de presión positiva. Estudios de mediano plazo

\begin{tabular}{lclccc}
\hline Autores (Ref) & $\begin{array}{c}\text { Presión, } \\
\mathbf{c m} \mathbf{H}_{\mathbf{2}} \mathbf{O}^{*}\end{array}$ & Diseño & Duración & $\begin{array}{c}\text { Número } \\
\text { Inician/Completan }\end{array}$ & $\begin{array}{c}\mathbf{P a C O}_{2}, \\
\mathbf{m m H g}^{\dagger}\end{array}$ \\
\hline Strumpf, $1991^{26}$ & $15 \pm 1 / 2$ & Cruzado & 3 meses & $19 / 7$ & $46 \pm 5$ \\
Gay, $1996^{24}$ & $10 / 2$ & Paralelo & 3 meses & $7 / 4$ & $55 \pm 9$ \\
Meecham Jones, $1995^{9}$ & $18 \pm 2 /$ & Cruzado & 3 meses & $18 / 14$ & $56 \pm 4$ \\
\hline
\end{tabular}

* Se describe presión inspiratoria/presión espiratoria. (Ref) = cita bibliográfica.

† Sólo se proporcionan los datos del grupo tratado con ventilación no invasiva.

Tabla 4. Ventilación no invasiva con ventiladores de presión positiva. Estudios de largo plazo

\begin{tabular}{llllcc}
\hline Autores (Ref) & $\begin{array}{c}\text { Presión, } \\
\mathbf{c m} \mathbf{H}_{\mathbf{2}} \mathbf{O}^{*}\end{array}$ & Diseño & Duración & $\begin{array}{c}\text { Número } \\
\text { Inician/Completan }\end{array}$ & $\begin{array}{c}\mathbf{P a C O}_{\mathbf{2}}, \\
\mathbf{m m H g}^{\dagger}\end{array}$ \\
\hline Casanova, $2000^{40}$ & $12 \pm 2 / 4$ & Paralelo & 1 año & $26 / 17$ & $51 \pm 8$ \\
Clini, $2002^{23}$ & $14 \pm 3 / 2 \pm 1$ & Paralelo & 2 años & $43 / 23$ & $54 \pm 5$ \\
\hline
\end{tabular}

* Se describe presión inspiratoria/presión espiratoria. (Ref) = cita bibliográfica.

† Sólo se proporcionan los datos del grupo tratado con ventilación no invasiva. 
De hecho, las características del sueño tendieron a empeorar con VNI. La Tabla 3 muestra diferencias en los niveles basales de $\mathrm{PaCO}_{2}$ entre los dos estudios, lo que se debe a que la hipercapnia fue criterio de inclusión sólo en el estudio de Meecham-Jones y cols ${ }^{19}$. También las presiones inspiratorias de este último estudio fueron mayores. Ello se explica porque estos autores emplearon la máxima presión tolerada y un período de aclimatación en el hospital para asegurar una adecuada tolerancia. En cambio, Strumpf y cols ${ }^{26}$, emplearon la presión inspiratoria necesaria para lograr una reducción arbitraria de la $\mathrm{PaCO}_{2}$ en $5 \mathrm{mmHg}$ y no hubo período de aclimatación.

Los resultados de Gay y $\operatorname{cols}^{24}$, fueron también desfavorables. Treinta y cinco pacientes hipercápnicos $\left(\mathrm{PaCO}_{2}>45 \mathrm{mmHg}\right)$ fueron incorporados para comparar VNI nocturna con VNI simulada. Después de un período de observación de 6 semanas sólo 13 fueron randomizados. La causa de exclusión más frecuente fue la normalización de la $\mathrm{PaCO}_{2}$ con terapia médica óptima. De no haber sido así, un número significativo no sólo habría recibido innecesariamente VNI, sino que podría haber sido erróneamente catalogado como beneficiado por ella. La presión inspiratoria se fijó en un valor muy bajo $\left(10 \mathrm{~cm} \mathrm{H}_{2} \mathrm{O}\right)$, sin que se proporcionen razones para ello, y no se empleó un período de adaptación en el hospital. Cuatro de los 7 pacientes asignados a VNI completaron el estudio y no mostraron beneficios en términos del intercambio de gases, caminata en 6 minutos ni calidad del sueño.

Ventiladores de presión positiva en estudios de largo plazo. Hasta la fecha sólo se han publicado dos ECR de duración prolongada (Tabla $4)^{23,40}$. El primero pertenece a Casanova y $\operatorname{cols}^{40}$, quienes asignaron aleatoriamente 52 pacientes a VNI o a tratamiento estándar. Doce pacientes tenían una $\mathrm{PaCO}_{2}$ mayor de $50 \mathrm{mmHg}$. Se empleó un período de aclimatación en el hospital. La presión de soporte inspiratorio se ajustó para disminuir el uso de los músculos accesorios, la sensación de disnea y reducir en un $20 \%$ la frecuencia respiratoria, sin proporcionarse justificación para estos criterios. Después de 12 meses de seguimiento la tasa de abandonos fue baja (16\%). Se observó una reducción significativa aunque marginal de la disnea (6 a 5 puntos según la escala del Medical Research Council [MRC]) y una mejoría de la coordinación psicomotora, efecto de valor clínico incierto proveniente de un set de pruebas neuro-psicológicas.
Sin embargo, no se apreciaron efectos significativos en el número de exacerbaciones, hospitalizaciones ni mortalidad. Por su parte, Clini y cols $^{23}$, llevaron a cabo un ensayo multicéntrico de dos años de duración en pacientes hipercápnicos ( $\mathrm{n}=90 ; \mathrm{PaCO}_{2}>50 \mathrm{mmHg}$ ), en que se comparó VNI con tratamiento estándar. Las presiones inspiratorias fueron mayores que en el estudio de Casanova y $\operatorname{cols}^{40}$, pero bajas comparadas con otros estudios ${ }^{19,30}$. Esto probablemente se explica porque se seleccionó la presión necesaria para lograr una reducción arbitraria de la $\mathrm{PaCO}_{2}$ en $5 \mathrm{mmHg}$ después de 1 hora de VNI. Se utilizó un período de adaptación en el hospital de 10 días y la adherencia fue muy elevada (87\%). No se observaron cambios en la función respiratoria, en la caminata en 6 minutos ni en las características del sueño al usar VNI. En cambio, hubo una mejoría en la calidad de vida y una disminución de la disnea según la escala MRC. Desafortunadamente estos últimos efectos son de interpretación incierta: el beneficio en la calidad de vida se apreció con el cuestionario MRF-28 (Maugeri Foundation Respiratory Failure), pero no con el de St. George's; mientras que la reducción de la disnea fue escasa, con una diferencia entre los grupos de apenas 0,6 puntos al final del estudio. Por último, el número de hospitalizaciones/año fue similar al igual que la mortalidad.

Revisiones sistemáticas y meta-análisis. Se ha publicado un meta-análisis ${ }^{32}$ y una revisión sistemática $^{41}$.

El meta-análisis fue publicado en 2003 y comprendió trabajos randomizados hasta el año 2000. Se incluyeron 4 ECR de buena calidad metodológica ${ }^{19,24,26,40}$, con un número total de 106 pacientes. Se concluyó que la aplicación nocturna de VNI no producía efectos significativos en la función respiratoria, en el intercambio de gases o en la eficiencia del sueño. Se apreció un aumento discreto de la fuerza muscular respiratoria, que los autores consideran clínicamente irrelevante, y un aumento promedio de la caminata en 6 minutos de 27,5 metros (IC 95\% -26,8 a 81,8). El amplio intervalo de confianza ha sido interpretado como sugerente de un efecto sustancial en un subgrupo de pacientes. Una limitante significativa de este estudio es el reducido número de pacientes incluido. Debido a la elevada tasa de abandonos en dos de los estudios y a los diferentes efectos evaluados, el número de pacientes disponibles para las comparaciones osciló entre 23 y 66.

La revisión sistemática, publicada en 2007, 
comprendió trabajos publicados hasta el año 2003. Se incluyeron 15 estudios realizados con ventiladores de presión positiva, 6 catalogados como ECR y 9 como no-ECR (cruzados), con un total de 466 pacientes. En comparación con el meta-análisis previo, se agregaron 2 ECR nuevos, uno de ellos fisiológico ${ }^{28}$ y otro con limitaciones metodológicas ${ }^{35}$. Sin embargo, dos estudios originalmente incluidos como ECR fueron reasignados en la revisión al grupo no-ECR ${ }^{19,26}$, siendo analizados en conjunto con una serie de trabajos que evaluaron efectos fisiológicos agudos de la VNI durante su aplicación en repo$\mathrm{so}^{33,34,42,43}$ o en ejercicio ${ }^{44}$. Como podría esperarse, las conclusiones obtenidas no difieren sustancialmente del meta-análisis. En relación a los ECR evaluados, los autores enfatizan que: a) la disnea disminuyó en 4 estudios; b) la calidad de vida mejoró en los dos estudios que la evaluaron; y c) los efectos sobre la frecuencia de hospitalizaciones han sido inconsistentes, mientras que la mortalidad no se ha modificado con la intervención. Concluyen que la VNI podría ser útil en pacientes con EPOC avanzada, hipercapnia e hiperinflación pulmonar dinámica. Un problema serio de esta revisión reside en el análisis conjunto de estudios marcadamente heterogéneos ${ }^{45}$.

En suma, la revisión sistemática y meta-análisis no proporcionan información consistente respecto a la utilidad de la VNI en los pacientes con EPOC avanzada.

\section{Efecto potencial}

La aplicación de VNI en pacientes con EPOC avanzada puede ser beneficiosa en algunos enfermos hipercápnicos, sintomáticos a pesar de tratamiento farmacológico óptimo, pero los estudios controlados aplicando apropiadamente la ventilación no invasiva son aún escasos y se requiere mayor evidencia para sustentar una recomendación.

\section{Evaluación del costo/beneficio}

De acuerdo a la información disponible, no es posible asegurar que la VNI en estos pacientes sea costo/efectiva. Para ello se requeriría demostrar un efecto sobre las hospitalizaciones y la mortalidad en estudios multicéntricos.

\section{Grado de recomendación}

La evidencia que sustenta el empleo de VNI en este grupo de enfermos es débil. Se basa en estudios de buena calidad metodológica, pero realizados en un número limitado de enfermos de características heterogéneas, con disparidad en la duración y los efectos evaluados, y con resultados contradictorios.

\section{Observaciones finales}

En los pacientes que permanecen marcadamente sintomáticos a pesar del tratamiento farmacológico óptimo, es muy probable que el médico tratante considere el empleo de la VNI como una herramienta terapéutica adyuvante. Por esta razón, aunque la recomendación es débil, se ha decidido proporcionar las siguientes consideraciones prácticas:

- Los pacientes que han mostrado los beneficios más evidentes son aquellos que poseen hipercapnia $\left(\mathrm{PaCO}_{2}\right.$ mayor de $\left.50 \mathrm{mmHg}\right)$.

- Aunque la hipercapnia se asocia estrechamente con el grado de hiperinflación pulmonar, no existe evidencia suficiente para sustentar la idea que la hiperinflación sea un criterio de selección.

- La VNI con ventiladores de presión negativa es mal tolerada y su empleo no se recomienda.

- Al ajustar el ventilador, debe tenerse en cuenta que los mayores efectos se han logrado usando las máximas presiones toleradas, generalmente mayor de $15 \mathrm{~cm} \mathrm{H}_{2} \mathrm{O}$. La presión de soporte inspiratorio no debería seleccionarse para lograr una reducción predefinida de la $\mathrm{PaCO}_{2}$ durante la VNI. El nivel apropiado de presión espiratoria se desconoce, pero en la mayor parte de los estudios ha oscilado entre 2-4 $\mathrm{cm} \mathrm{H}_{2} \mathrm{O}$.

- La VNI puede aplicarse tanto durante el día como en la noche. Si la aplicación diurna se realiza en un ambiente hospitalario garantiza una mejor vigilancia del uso apropiado del ventilador, pero puede resultar difícil de implementar. Por el contrario, la aplicación nocturna es más fácil de implementar para uso domiciliario, pero no asegura que se logre un apropiado uso del ventilador.

- Todos los estudios en que la VNI ha sido beneficiosa han utilizado un período de aclimatación en el hospital. Esto puede realizarse con el paciente hospitalizado o en forma ambulatoria en un laboratorio de función pulmonar.

- El número de horas de uso diario se desconoce. Cuando se ha utilizado en ambientes vigilados, 2-3 horas parecen suficientes para lograr beneficios clínicos sustanciales. Si se utiliza durante la noche debería aplicarse el máximo de horas posible. No existe ningún método que permita evaluar satisfactoriamente la apropiada ventilación en el domicilio. 


\section{Bibliografía}

1.- BROCHARD L. Non-invasive ventilation for acute exacerbations of COPD: a new standard of care. Thorax 2000; 55: 817-8.

2.- MEHTA S, HILL N S. Noninvasive ventilation. Am J Respir Crit Care Med 2001; 163: 540-77.

3.- Clinical indications for noninvasive positive pressure ventilation in chronic respiratory failure due to restrictive lung disease, COPD, and nocturnal hypoventilation. A Consensus Conference Report Chest 1999;116:521-534.

4.- BTS GUIDELINE B. Non-invasive ventilation in acute respiratory failure. Thorax 2002; 57: 192-211.

5.- MACKLEM P. The clinical relevance of respiratory muscle research: J Burns Amberson Lecture. Am Rev Respir Dis 1986; 134: 812-5.

6.- AMBROSINO N, MONTAGNA T, NAVA S, NEGRI A, BREGA S, FRACCHIA C, et al. Short term effect of intermittent negative pressure ventilation in COPD patients with respiratory failure. Eur Respir J 1990; 3: $502-8$

7.- BRAUN N, MARINO W. Effect of daily intermittent rest of respiratory muscles in patients with severe chronic airflow limitation. Chest 1984; 85: 59-60 (S).

8.- CROPP A, DIMARCO A. Effects of intermittent negative pressure ventilation on respiratory muscle function in patients with severe chronic obstructive pulmonary disease. Am Rev Respir Dis 1987; 135: 1056-61.

9.- GUTIÉRREZ M, BEROÍZA T, CONTRERAS G, DÍAZ O, CRUZ E, MORENO R, et al. Weekly cuirass ventilation improves blood gases and inspiratory muscle strength in patients with chronic air-flow limitation and hypercarbia. Am Rev Respir Dis 1988; 138: $617-23$

10.- SCANO G, GIGLIOTTI F, DURANTI R, SPINELLI A, GORINI M, SCHIAVINA M. Changes in ventilatory muscle function with negative pressure ventilation in patients with severe COPD. Chest 1990; 97 (2): 3227.

11.- CELLI B, LEE H, CRINER G, BERMUDEZ M, RASSULO J, GILMARTIN M, et al. Controlled trial of external negative pressure ventilation in patients with severe chronic airflow obstruction. Am Rev Respir Dis 1989; 140: 1251-6.

12.- SHAPIRO S, ERNST P, GRAY-DONALD C, MARTIN J, WOOD-DAUPHINEE S, BEAUPRÉ A, et al. Effect of negative pressure ventilation in severe chronic obstructive pulmonary disease. Lancet 1992; 340: 1425-9.

13.- ZIBRAK J, HILL N, FEDERMAN E, KWA S, O`DONNELL C. Evaluation of intermittent long-term negative-pressure ventilation in patients with severe chronic obstructive pulmonary disease. Am Rev Respir Dis 1988; 138: 1515-8.

14.- SIMILOWSKI T, YAN S, GAUTHIER A P, MACKLEM P T, BELLEMARE F. Contractile properties of the human diaphragm during chronic hyperinflation. N Engl J Med 1991; 325: 917-23.

15.- BEGIN P, GRASSINO A. Inspiratory muscle dysfunction and chronic hypercapnia in chronic obstructive pulmonary disease. Am Rev Respir Dis 1991; 143: 905-12.

16.- CATTERALL J, DOUGLAS N, CALVERLEY P, SHAPIRO C, BREZINOVA V, BRASH $\mathrm{H}$, et al. Transient hypoxemia during sleep in chronic obstructive pulmonary disease is not a sleep apnea syndrome. Am Rev Respir Dis 1983; 128: 24-9.

17.- FLEETHAM J, WEST P, MEZON B, CONWAY W, ROTH T, KRYGER M. Sleep, arousals and oxygen desaturation in chronic obstructive pulmonary disease. Am Rev Respir Dis 1982; 126: 429-33.

18.- ELLIOTT M W, MULVEY D A, MOXHAM J, GREEN M, BRANTHWAITE M A. Domiciliary nocturnal nasal intermittent positive pressure ventilation in COPD: mechanisms underlying changes in arterial blood gas tensions. Eur Respir J 1991; 4: 1044-52.

19.- MEECHAM JONES D J, PAUL E A, JONES P W, WEDZICHA J A. Nasal pressure support ventilation plus oxygen compared with oxygen therapy alone in hypercapnic COPD. Am J Respir Crit Care Med 1995; 152: $538-44$.

20.- TURKINGTON P M, ELLIOTT M W. Rationale for the use of non-invasive ventilation in chronic ventilatory failure. Thorax 2000; 55: 417-23.

21.- JUAN G, CALVERLEY P, TALAMO C, SCHNADER J, ROUSSOS C. Effect of carbon dioxide on diaphragmatic function in human beings. N Engl J Med 1984; 310: 874-9.

22.- CLOOSTERMAN S G M, HOFLAND I D, VAN SCHAYCK C P, FOLGERING H T M. Exertional dyspnoea in patients with airway obstruction, with and without CO2 retention. Thorax 1998; 53: 76874.

23.- CLINI E, STURANI C, ROSSI A, VIAGGI S, CORRADO A, DONNER C F, et al. The Italian multicentre study on noninvasive ventilation in chronic obstructive pulmonary disease patients. Eur Respir J 2002; 20: 529-38.

24.- GAY P, HUBMAYR R, STROETZ R. Efficacy of nocturnal nasal ventilation in stable, severe chronic obstructive pulmonary disease during a 3-month controlled trial. Mayo Clin Proc 1996; 71: 533-42.

25.- LIN C C. Comparison between nocturnal nasal positive pressure ventilation combined with oxygen therapy and oxygen monotherapy in patients with severe COPD. Am J Respir Crit Care Med 1996; 154: 353-8.

26.- STRUMPF D, MILLMAN R, CARLISLE C, GRATTAN L, RYAN S, ERICKSON A, et al. Nocturnal positive-pressure ventilation via nasal mask in patients with severe chronic obstructive pulmonary disease. Am Rev Respir Dis 1991; 144: 1234-9.

27.- HALUSZKA J, CHARTRAND D, GRASSINO A, MILIC-EMILI J. Intrinsic PEEP and arterial $\mathrm{PCO}_{2}$ in stable patients with chronic obstructive pulmonary disease. Am Rev Respir Dis 1990; 141: 1194-7.

28.- DÍAZ O, BEGIN P, TORREALBA B, JOVER E, LISBOA C. Effects of noninvasive ventilation on lung hyperinflation in stable hypercapnic COPD. Eur Respir J 2002; 20: 1490-8.

29.- INGENITO E P, LORING S H, MOY M L, MENTZER $\mathrm{S}$ J, SWANSON S J, REILLY J J. Interpreting improvement in expiratory flows after lung volume reduction surgery in terms of flow limitation theory. Am J Respir Crit Care Med 2001; 163: 1074-80.

30.- DÍAZ O, BEGIN P, ANDRESEN M, PRIETO M E, CASTILLO C, JORQUERA J, et al. Physiological and clinical effects of diurnal noninvasive ventilation in hypercapnic COPD. Eur Respir J 2005; 26: 1016-23.

31.- BUDWEISER S, HEINEMANN F, FISCHER W, DOBROSCHKE J, PFEIFER M. Long-term reduction of hyperinflation in stable COPD by non-invasive nocturnal home ventilation. Respir Med 2005; 99: 976-84. 
32.- WIJKSTRA P J, LACASSE Y, GUYATT G H, CASANOVA C, GAY P C, MEECHAM JONES J, et al. A meta-analysis of nocturnal noninvasive positive pressure ventilation in patients with stable COPD. Chest 2003; 124: 337-43.

33.- NAVA S, AMBROSINO N, RUBINI F, FRACCHIA C, RAMPULLA C, TORRI G, et al. Effect of nasal pressure support ventilation and external PEEP on diaphragmatic activity in patients with severe stable COPD. Chest 1993; 103: 143-50.

34.- AMBROSINO N, NAVA S, BERTONE P, FRACCHIA C, RAMPULLA C. Physiologic evaluation of pressure support ventilation by nasal mask in patients with stable COPD. Chest 1992; 101: 385-91.

35.- GARROD R, MIKELSONS C, PAUL E A, WEDZICHA J A. Randomized controlled trial of domiciliary noninvasive positive pressure ventilation and physical training in severe chronic obstructive pulmonary disease. Am J Respir Crit Care Med 2000; 162: 1335-41.

36.- KRACHMAN S L, QUARANTA A J, BERGER T J, CRINER G J. Effects of noninvasive positive pressure ventilation on gas exchange and sleep in COPD patients. Chest 1997; 112: 623-8.

37.- GIGLIOTTI F, SPINELLI A, DURANTI R, GORINI M, GOTI P, SCANO G. Four-week negative pressure ventilation improves respiratory function in severe hypercapnic COPD patients. Chest 1994; 105: 8794.

38.- NAVA S, FANFULLA F, FRIGERIO P, NAVALESI P. Physiologic evaluation of 4 weeks of nocturnal nasal positive pressure ventilation in stable hypercapnic patients with chronic obstructive pulmonary disease. Respiration 2001; 68: 573-83.

39.- RENSTON J P, DIMARCO A F, SUPINSKI G S. Respiratory muscle rest using nasal BiPAP ventilation in patients with stable severe COPD. Chest 1994; 105 : 1053-60.

40.- CASANOVA C, CELLI B R, TOST L, SORIANO E, ABREU J, VELASCO V, et al. Long-term controlled trial of nocturnal nasal positive pressure ventilation in patients with severe COPD. Chest 2000; 118: 158290.

41.- KOLODZIEJ M A, JENSEN L, ROWE B, SIN D. Systematic review of noninvasive positive pressure ventilation in severe stable COPD. Eur Respir J 2007; 30: 293-306.

42.- LIEN T C, WANG J H, CHANG MT, KUO C D. Comparison of BiPAP nasal ventilation and ventilation via iron lung in severe stable COPD. Chest 1993; 104: 460-6.

43.- MARANGONI S, VITACCA M, QUADRI A, SCHENA M, CLINI E. Non-invasive haemodynamic effects of two nasal positive pressure ventilation modalities in stable chronic obstructive lung disease patients. Respiration 1997; 64: 138-44.

44.- HIGHCOCK M P, SHNEERSON J M, SMITH I E. Increased ventilation with NiIPPV does not necessarily improve exercise capacity in COPD. Eur Respir J 2003; 22: 100-5.

45.- DUIVERMAN M L, STRUIK F M, WIJKSTRA P J. Noninvasive ventilation in severe stable COPD: is it effective, and if so, in what way? Eur Respir J 2008; 31: 1136-7.

Correspondencia a:

Dr. Orlando Díaz P.

E-mail: odiazp@vtr.net 